\section{LA CLAVE ES EL PAISAJE. EXPLORANDO ALTERNATIVAS AL TURISMO DE MASAS}

\author{
Raquel Huete \\ Universidad de Alicante \\ ORCID iD: http://orcid.org/0000-0001-5576-1614 \\ r.huete@ua.es \\ Alejandro Mantecón \\ Universidad de Alicante \\ ORCID iD: http://orcid.org/0000-0002-3208-2114 \\ alejandro.mantecon@ua.es
}

Cómo citar este artículo/Citation: Huete, R. y Mantecón, A. (2017). La clave es el paisaje. Explorando alternativas al turismo de masas. Arbor, 193 (785): a397. doi: http://dx.doi. org/10.3989/arbor.2017.785n3003

Recibido: 21-09-2016. Aceptado: 25-6-2017.

RESUMEN: Este texto propone una reflexión sobre la posibilidad de desarrollar proyectos innovadores de turismo sostenible en torno al concepto de paisaje en contextos que han experimentado procesos de masificación turística estrechamente ligados a la actividad inmobiliaria. Tomando como marco de referencia la provincia de Alicante, ejemplo ilustrativo de región especializada en la vertiente residencial, se identifica como caso de estudio paradigmático el proyecto Vivood Landscape Hotel, cuyo entorno inmediato lo conforma el municipio de Benimantell y el valle de Guadalest, a pocos kilómetros de Benidorm. La reflexión se fundamenta en una investigación de carácter cualitativo basada principalmente en la aplicación de entrevistas en profundidad a actores sociales clave a propósito de esta iniciativa empresarial multipremiada, en la que convergen criterios de sostenibilidad arquitectónica y ambiental con un desarrollo integrador y respetuoso con los intereses socioeconómicos de la población local.

PALABRAS CLAVE: sostenibilidad; medio ambiente; innovación; hoteles; Benimantell; Alicante.

\section{LANDSCAPE IS THE KEY. EXPLORING MASS TOURISM ALTERNATIVES}

Copyright: (C) 2017 CSIC. Este es un artículo de acceso abierto distribuido bajo los términos de la licencia Creative Commons Attribution (CC BY) España 3.0.

ABSTRACT: We offer a reflection on the possibility of developing innovative sustainable tourism projects around the concept of landscape in environments linked to mass tourism and real estate development. In the framework of the province of Alicante, an illustrative example of a region specializing in residential tourism, the project Vivood Landscape Hotel is identified as a paradigmatic case, whose immediate environment is the municipality of Benimantell and Guadalest Valley, a few kilometres from Benidorm. This reflection is based on qualitative research based primarily on the application of in-depth interviews with key stakeholders regarding this multiawarded entrepreneurship. Here, criteria of architectural and environmental sustainability are combined with a development that is inclusive and respectful with the socio-economic interests of the local population.

KEYWORDS: sustainability; environment; innovation; hotels; Benimantell; Alicante. 


\section{INTRODUCCIÓN}

La Comunidad Valenciana es el tercer destino turístico de España (por detrás de Cataluña y Andalucía) en número de viajes nacionales y extranjeros (casi 23 millones) y de pernoctaciones (156 millones) (datos de Frontur-Egatur y ETR-Familitur para 2015). La posición desciende hasta el quinto puesto si se considera únicamente a los turistas extranjeros. Sin embargo, al observar las cifras de alojamiento en su propia residencia ocupa el primer puesto para el turismo extranjero (20 millones) y el segundo tanto para el turismo nacional (19,7 millones de pernoctaciones) como para quienes se hospedan en viviendas de familiares o amigos. De hecho, de los 6,5 millones de turistas extranjeros que la Comunidad Valenciana recibió en 2015: 1,2 millones se alojó en vivienda propia, 1,8 en vivienda de familiares y amigos y 1,1 millones en vivienda de alquiler (Agencia Valenciana de Turismo, estimación anual a partir de Frontur-Egatur, cuota acumulada a septiembre).

Específicamente, en la provincia de Alicante llama la atención el modo en el que los flujos turístico-residenciales y la inmigración de ciudadanos europeos procedentes de países con una renta per cápita más alta que la española se ha adaptado a la oferta inmobiliaria generada por la masiva construcción de viviendas edificadas sobre todo en las periferias de los centros urbanos tradicionales, en un principio destinadas a un uso no principal (Membrado Tena, 2013; Membrado Tena, 2014). El modo de producción que se sustenta en el binomio construcción-ocio halla su origen en Alicante durante los años sesenta, y en particular en la década de 1970, cuando se ocupan grandes espacios en los municipios costeros justificando la necesidad de explotar turísticamente las playas. En los ochenta, el proceso se extiende a municipios distantes de la costa entre 5 y 20 kilómetros, aprovechando la mejora de accesos, la fuerte demanda y el menor coste del suelo para, a finales de los noventa, alcanzar a las poblaciones del interior debido al precio del suelo aún más bajo y a la pérdida de calidad motivada por la saturación urbanística y la degradación ambiental de los municipios de primera y segunda línea (Huete, 2009).

Los esfuerzos llevados a cabo durante el presente siglo con el propósito de diversificar la oferta turística de la región y adaptarla a un contexto postfordista se han visto constreñidos de diversas formas por las inercias preexistentes, extendidas hasta el paroxismo a lo largo de la conurbación litoral: aproximadamente un $65 \%$ del frente litoral valenciano se corresponde con suelo urbano consolidado, ocupado con tipologías en su mayoría residenciales.
Sin olvidar las tendencias dominantes, en las siguientes páginas se invita a adoptar una posición de optimismo muy comedido. En concreto, se trata de mostrar cómo, en un contexto determinado por la exacerbación de las inercias inmobiliarias y la instrumentalización de la naturaleza por los intereses de la industria del ladrillo, pueden impulsarse proyectos alternativos al turismo de masas que contribuyan a mejorar la calidad y la imagen de los destinos y, en consecuencia, que favorezcan la llegada de turistas más exigentes, respetuosos y con una mayor capacidad de gasto. Bajo ese objetivo general se reconoce un objetivo más concreto relacionado con la identificación y comprensión de las dificultades inherentes a la implementación de un proyecto de estas características y de los riesgos que lo amenazan. Con esta intención se plantea un texto dividido en varias partes: primero, se esboza una reflexión acerca de las relaciones entre la valorización del paisaje no antropizado como recurso turístico y su utilidad para la innovación empresarial; a continuación se explica el enfoque metodológico que orienta el estudio, sustentado en el análisis de "un caso paradigmático": el del Hotel Paisaje Vivood; en tercer lugar, se presenta un argumento analítico a la luz de los hallazgos obtenidos; y, finalmente, en las conclusiones se discuten algunas cuestiones derivadas de los apartados anteriores.

\section{INNOVACIÓN Y PAISAJE}

El concepto de innovación es escurridizo pues resulta difícil acotar qué aspectos particulares de una realidad pueden catalogarse como innovadores, y más en un sector tan dinámico como el turístico (Hall y Williams, 2008). A partir del trabajo de Rodríguez, Williams y Hall (2014) sobre la innovación en la política turística española podría aceptarse como punto de partida que la innovación en el ámbito empresarial se sostiene sobre dos elementos: la creatividad, entendida aquí como la facultad de inventar una respuesta novedosa para abordar un desafío concreto, y la implementación, o sea, la viabilidad asociada a la aplicación práctica de esa respuesta o, dicho de otro modo, el hecho de que la novedad de la respuesta (creación o mejora de un producto, cambio en una estructura organizativa, apertura de un nuevo mercado, etc.) se convierta en una acción que genere rentabilidad. En su aproximación al análisis de la innovación en el sistema turístico de la provincia de Alicante, Rodríguez (2016) presenta una tipología de ejemplos de innovación en tres bloques: 1) el producto, 2) la gestión y 3) los instrumentos organizativos de la política turística regional. A su vez, en el primero de los bloques cita- 
dos distingue varios apartados. Para los objetivos del presente trabajo, interesa resaltar que en el marco de la innovación en establecimientos hoteleros la autora identifica el Hotel Paisaje Vivood como un caso paradigmático de nuevo concepto de negocio innovador basado en la exclusividad. De este modo, se empieza a justificar su selección como objeto de estudio.

El paisaje, elemento auténticamente distintivo del Vivood, es otro concepto "borroso". Desde una perspectiva a medio camino entre la Sociología y las Humanidades, podría acordarse que el paisaje en una construcción histórica y social ligada a criterios estéticos subjetivos que normalmente se forman después de vivir y comparar lugares con rasgos diferentes (Roger, 1997). Es decir, los campesinos no crean el ideal romántico de la naturaleza. En cambio, este surge en el siglo XIX al experimentar esa naturaleza poetas y viajeros procedentes de las ciudades, hastiados de enfrentarse a la realidad urbano-industrial en la que cristalizaba el proyecto ilustrado. Para el campesino de la Provenza el paisaje que pinta Van Gogh es el telón de fondo de su vida cotidiana, asociado en primer lugar a la dureza de un medio en el que discurren los trabajos del campo. En términos literarios, diríase que la belleza habita no tanto en el objeto admirado como en los ojos de quien lo admira. Desde este prisma, y más allá de las estrategias de marketing, la decisión de singularizar un establecimiento hotelero mediante su catalogación como "Hotel Paisaje" resulta problemática, pues al mirar desde la ventana de un hotel es muy habitual poder ver un paisaje, aunque sea el paisaje enladrillado de una hilera de edificios. En realidad, lo que se propone es un juego mental, que se mantiene en un nivel latente, por el cual la etiqueta "Hotel Paisaje" se vincula con un alojamiento que permite acceder a unas vistas antagónicas a aquellas que envuelven el medio urbano en el que se desarrolla el día a día de los potenciales huéspedes. El paisaje lo es en tanto que constituye una realidad escasamente antropizada, constituida naturalmente en forma de montañas, estepas, desiertos, lagos o playas. Es en la experiencia del contraste donde reside la emoción y es precisamente la expectativa de emocionarse lo que atrae a nuevos perfiles de turistas (Cohen, 2004; Cohen, 2005), en este caso susceptibles de conformar un segmento de la demanda que se define por el deseo de aprovechar el tiempo que dedica a hacer turismo para aproximarse a la naturaleza desde el respeto y la voluntad de preservación de la misma.

De este modo, el paisaje no violentado por la acción humana y diferenciado de los paisajes urbanos pre- dominantes en las ciudades -escenarios de las rutinas diarias, paulatinamente congestionadas por el tráfico y degradadas por la contaminación atmosférica y acústica- se torna en recurso turístico de gran valor para un sector del mercado, en ascenso durante las últimas dos décadas, que demanda experiencias alejadas de aquellas otras que caracterizan los mercados masivos del turismo fordista emergentes en los centros históricos del capitalismo industrial tras la Segunda Guerra Mundial.

\section{METODOLOGÍA}

La investigación se presenta como un estudio de caso de carácter cualitativo sustentado en el comentario analítico de datos básicos y, fundamentalmente, en la aplicación de cinco entrevistas en profundidad a una serie de informantes clave. Por lo tanto, no se persigue concluir con la formalización de un modelo teórico o estableciendo generalizaciones. Solo se busca completar una aproximación analítica al entendimiento de una determinada realidad. El caso en cuestión se corresponde con el proyecto del Hotel Paisaje Vivood. Su presentación así como la justificación metodológica de su selección confluyen con el análisis propiamente dicho y por eso se aborda directamente al inicio del siguiente apartado. Las cinco personas entrevistadas fueron Daniel Mayo, arquitecto y director general del Vivood (se apunta su identidad privada porque es el único de los entrevistados que dio su consentimiento explícito para que así se hiciera), tres empleados de la empresa con distintos perfiles que, al mismo tiempo, son residentes en municipios muy próximos al hotel $y$, en consecuencia, tienen formada una impresión de primera mano acerca de la percepción que del proyecto se tiene en las poblaciones más cercanas (se trata de una camarera, un técnico de mantenimiento responsable de trabajos selvícolas y una persona con responsabilidades administrativas) y un político representante del gobierno local en el Ayuntamiento de Benimantell, en cuyo término municipal se emplaza el hotel. El trabajo de campo se realizó durante el mes de julio de 2016. Todas las entrevistas tuvieron lugar en las instalaciones del hotel a excepción de la entrevista al representante del ayuntamiento, que se llevó a cabo en las dependencias de la empresa que dirige. La duración media de las entrevistas fue de cuarenta y cinco minutos. Tras la grabación de las mismas se procedió a su transcripción íntegra. El tipo de entrevista realizada se corresponde con lo que en la bibliografía anglosajona se denomina entrevista no estandarizada (Nonstandardized o Unstandardized interview), en la que únicamente se plantea un tema general y las pre- 
guntas abiertas específicas surgen a medida que se profundiza y avanza con la propia entrevista (Monette, Sullivan y DeJong, 2005). Es más frecuente utilizar estrategias entrevistadoras intermedias entre la estandarización y la opción aquí tomada, pero teniendo en cuenta cuáles eran los objetivos y el hecho de que en este caso se contaba con un entrevistador experimentado en la investigación del turismo desde enfoques cualitativos, se pensó que lo más aconsejable era actuar de este modo. El análisis de los textos transcritos no pretendía tanto identificar patrones discursivos sobre distintos temas susceptibles de ser comparados entre sí, pues sería absurdo con una muestra tan pequeña, sino, más bien, identificar claves explicativas útiles para dar forma a una narración documental capaz de responder al objetivo propuesto: entender cómo es posible crear un producto turístico innovador propio de un modelo turístico postfordista en un contexto -el de la provincia de Alicante- caracterizado por la hegemonía de un modelo turístico fordista masivo orientado intensamente por la función inmobiliarioresidencial (Huete, 2016). Al respecto, se ha decidido desplegar el argumento analítico prescindiendo de la integración en el texto de posible verbatim.

\section{ANÁLISIS}

\subsection{Presentación del caso: localización, historia y ca- racterísticas del Hotel Paisaje Vivood}

En el mapa 1 (figura 1) se representa la ubicación del Vivood, en el término municipal del Benimantell (con una población de 501 habitantes según la revisión del padrón municipal a 1 de enero de 2015), a 62 kilómetros de Alicante y en pleno Valle de Guadalest, entre las sierras de la Serrella y de Aitana (máxima altura de la provincia de Alicante con 1.558 metros), a 22 kilómetros de Benidorm en la costa y a 36 kilómetros de Alcoy en el interior.

Figura 1. Localización del Vivood.

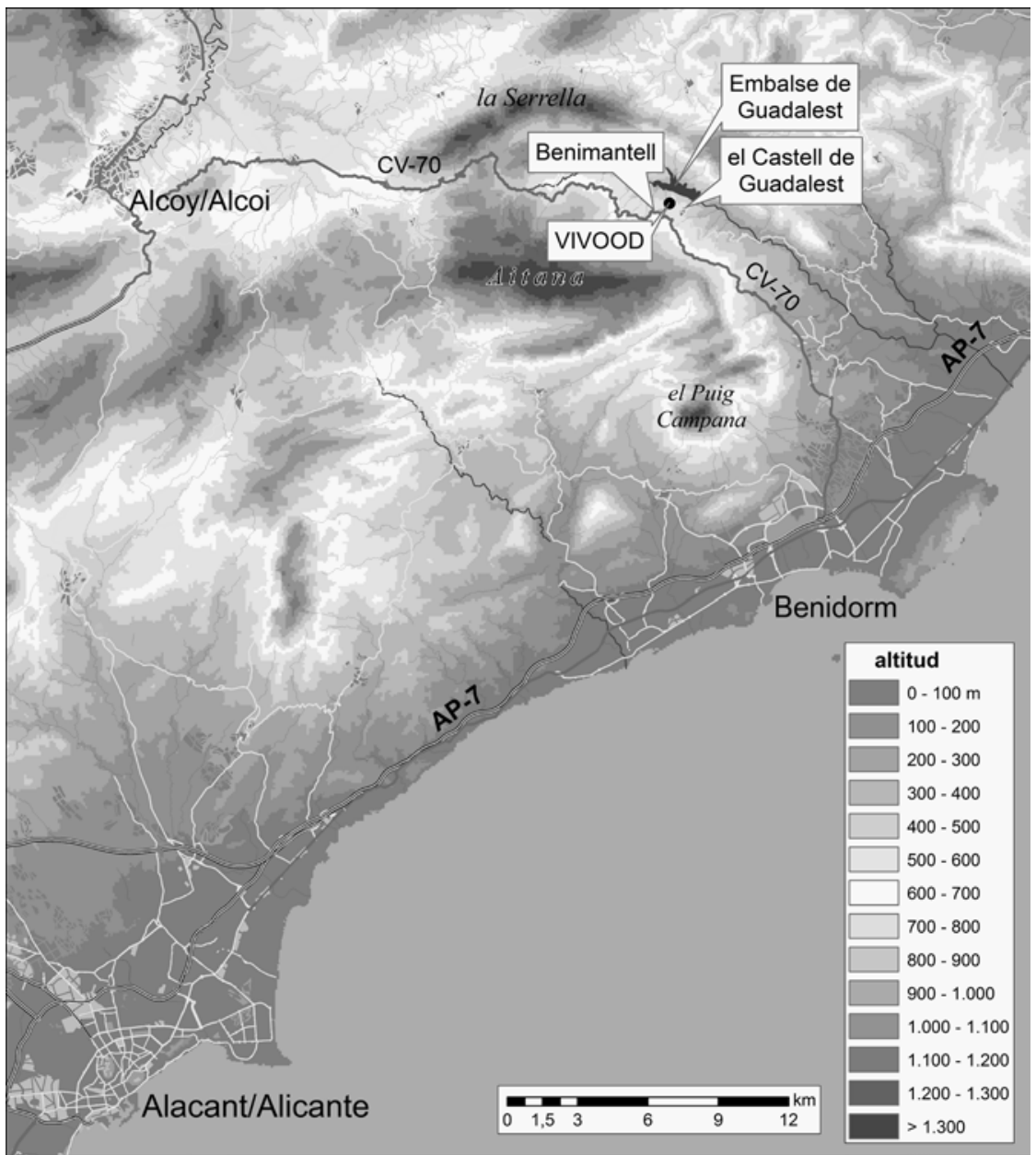


El Vivood abrió sus puertas al público en junio de 2015, pero la prehistoria de este hotel hunde sus raíces unos seis años antes, en una parcela de Aranjuez (Comunidad de Madrid) donde Daniel Mayo y Álvaro Figueruelo, entonces estudiantes de Arquitectura, intentaban construir una vivienda de 5 por 5 por 5 metros, destinada a ser una casa de fardos de paja prensados con gran capacidad de aislamiento, en la que invirtieron los 2.000 euros que habían obtenido al ganar un premio académico. El dinero se acabó y comenzaron a presentarse, y a ganar, otros concursos de los que se servían para financiar su novedosa casa sostenible. Entre ellos destaca el primer premio de la III Edición del Concurso Internacional de Diseño Talentos Design 11, auspiciado por la Fundación Banco Santander, con un proyecto que reconvertía su vivienda sostenible en un prototipo de alojamiento de emergencia autoconstruible en zonas afectadas por desastres naturales. Después de cuatro años adaptando su propuesta habitacional a la realidad de un contexto post-catástrofe natural, formalizaron todo el trabajo en la redacción de una investigación que, en 2011, mereció el primer premio en la sección de Arquitectura del X Certamen Universitario Arquímedes de Introducción a la Investigación Científica (Ministerio de Educación). Sin embargo, su creativo producto no pudo convertirse en una aportación auténticamente innovadora pues la implementación no tuvo éxito desde el punto de vista de la rentabilidad empresarial. Básicamente, les resultó imposible introducirse en los exigentes mercados internacionales en los que se comercializan las viviendas post-catástrofe. En ese momento Figueruelo reorienta sus intereses profesionales y Mayo decide continuar con el proyecto, aunque redefiniéndolo completamente. Es entonces cuando entra en contacto con el concepto glamping.

El glamping (neologismo formado a partir de la unión de glamour y camping) es una propuesta alternativa al camping tradicional que incorpora comodidades propias de establecimientos más sofisticados en alojamientos localizados en entornos naturales. Los principios del glamping se adaptaban bastante bien al desarrollo de la vivienda con la que Mayo llevaba tanto tiempo trabajando. El glamping se basa en proporcionar comodidades en entornos naturales alejados de la civilización que, realmente, comparten algunos rasgos fundamentales con una zona devastada por una catástrofe: escasea el agua, no hay luz, la logística desempeña un papel crucial y, en definitiva, se requieren unos sistemas de construcción de alojamiento muy peculiares. Así, empieza a ajustar su modelo. Vuelve a presentarse a diversos concursos y acaba siendo seleccionado por el programa Lanzadera de apoyo a emprendedores promovido desde Valencia por Juan Roig (presidente y máximo accionista de Mercadona).

La propuesta de Mayo fue una de las quince seleccionadas entre las 4.200 presentadas en la edición de 2013 y recibió una inversión inicial de 200.000 euros. De esta manera, se traslada a Valencia con un proyecto que consistía en vender casitas de madera desmontables, fácilmente transportables y autoconstruibles para campings interesados en proponer una oferta glamping y para casas rurales que quisieran ampliar su ocupación. Contaba con el respaldo financiero y el asesoramiento de los expertos del programa Lanzadera. Daba la sensación de que por fin todo funcionaba, no obstante Mayo no se sentía plenamente satisfecho con las inercias que se estaban fraguando: se había convertido en un vendedor de casas, pero no controlaba el uso final que los consumidores le daban al producto que había creado y las connotaciones distintivas de su idea, estrechamente ligadas a elementos subjetivos y experienciales, tendían a diluirse.

Mayo decide dar un último giro y, mientras desde Lanzadera desarrolla el proyecto seleccionado, prepara una variación importante del modelo de negocio previsto. Comprueba que incorporando elementos de lujo y exclusividad podía saltar del glamping a un formato aún más minoritario: el Landscape Hotel, y se propone rediseñar su estructura de alojamiento para construir y dirigir él mismo un Hotel Paisaje de referencia en España. Tras persuadir al fondo de inversión de la conveniencia de adoptar este nuevo enfoque, y unos meses antes de completar su estancia en el programa Lanzadera, inicia una exhaustiva búsqueda de posibles emplazamientos que acaba en Benimantell, aprovechando una parcela de $84.000 \mathrm{~m}^{2}$ donde años antes se había ubicado un centro de rescate y protección de animales.

Las veinticinco suites independientes que hoy constituyen la oferta de alojamiento del Vivood se integran en un entorno natural en el que se une el bosque mediterráneo con laderas de terrazas de época musulmana. La reducida escala de cada uno de los volúmenes independientes que componen el hotel facilita su integración paisajística y la conservación de las especies vegetales preexistentes. El proyecto implicó la plantación de especies autóctonas en las áreas originalmente despobladas, el control de otras de tipo invasivo y el desarrollo de un metódico plan de preservación ambiental. La ejecución de las edificaciones como módulos construidos en taller evitó arrojar es- 
combros, lo que redujo la duración de la obra y la contaminación acústica. Su llamativa disposición como estructuras independientes y elevadas sobre un sistema de cimentación reversible mediante pilares metálicos ha permitido mantener la topografía del terreno.
El sistema de iluminación indirecta empleado reduce la contaminación lumínica. Los dispositivos dispuestos son de tecnología Led, lo que disminuye el consumo energético, elimina los focos de calor y, con ello, aminora el riesgo de incendios. Cuenta con una

Figura 2. Perspectiva de los módulos que componen el Vivood.

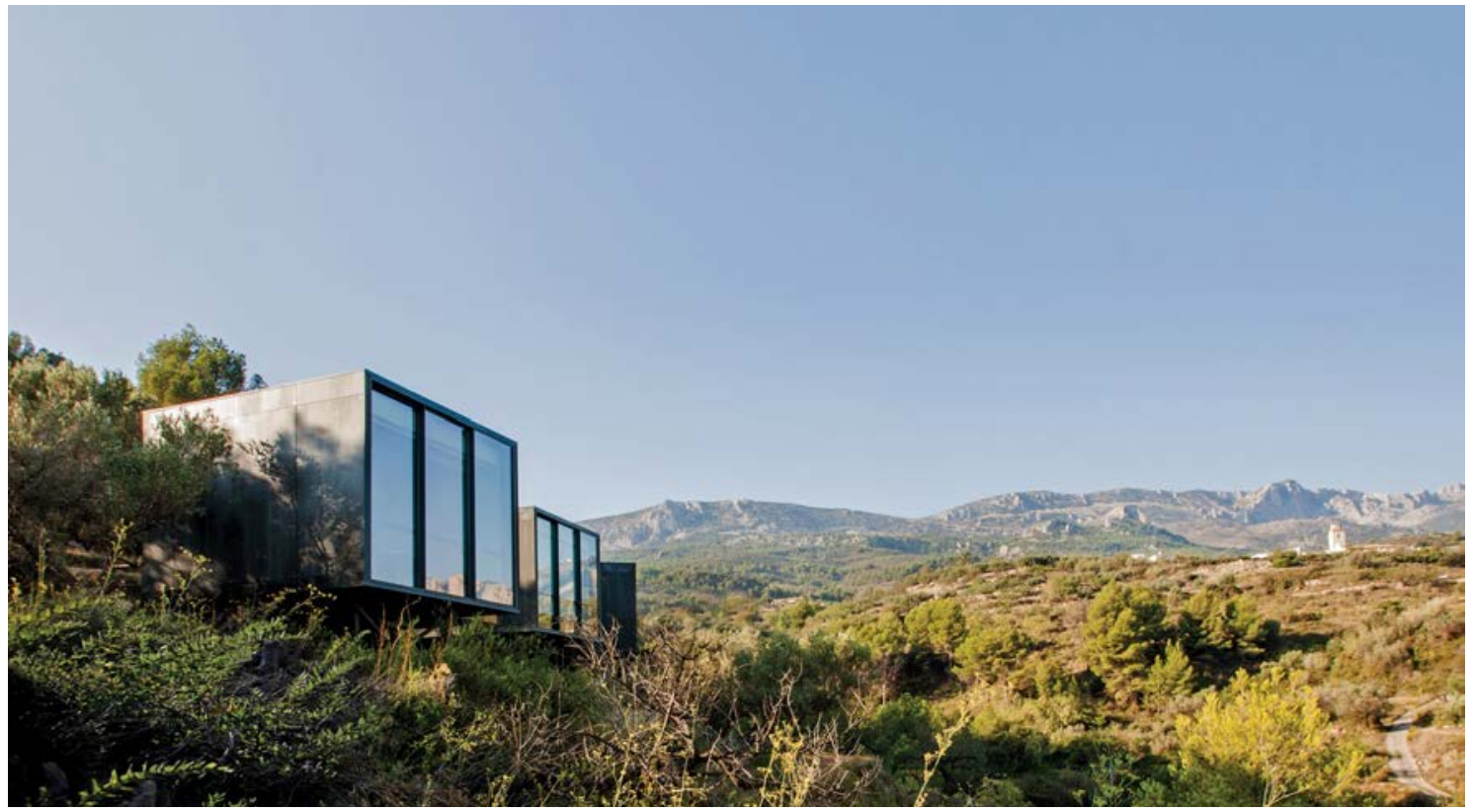

Fuente: Hotel Paisaje Vivood.

Figura 3. Imagen posterior de los módulos que componen el Vivood.

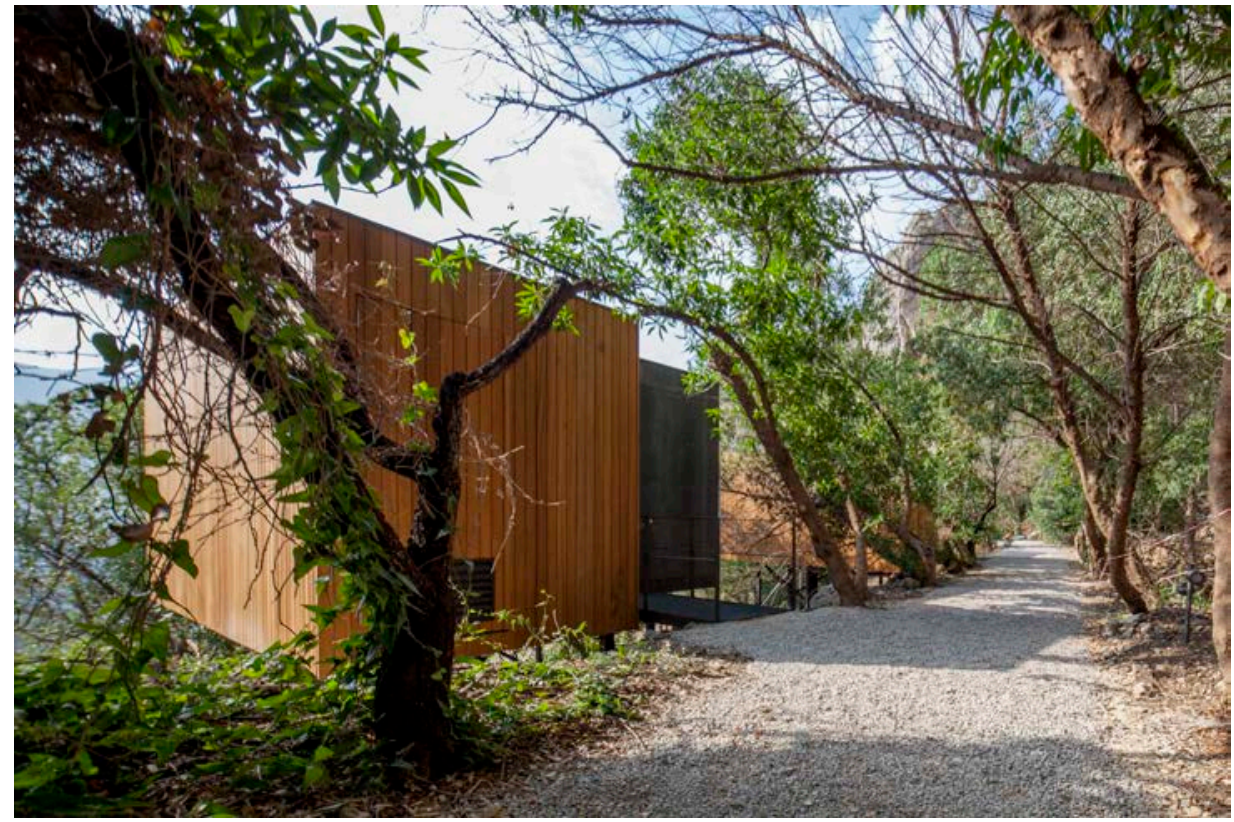

Fuente: Hotel Paisaje Vivood. 
superficie de placas fotovoltaicas que ofrece aporte energético extra. Las luminarias destinadas a señalizar los accesos al hotel se alimentan exclusivamente de energía solar. Los vehículos empleados en el recinto son eléctricos. Además, se emplean sistemas de gestión eficiente del consumo del agua y del mantenimiento de la piscina y los jacuzzis.

Se fomenta la contratación de personal residente en la zona con el fin de dinamizar el desarrollo local. La plantilla del Vivood, al redactar estas páginas, está integrada por veintiuna personas (once mujeres y diez hombres) de edades comprendidas entre los 21 y los 57 años, si bien, diecisiete (el 80\%) no superan los 33 años. Doce de los empleados residen en el Valle de Guadalest. Además, se cuenta con la colaboración puntual de profesionales residentes en el valle o en sus inmediaciones (profesores de yoga, masajistas, expertos en reiki, etc.). Acorde con principios de sostenibilidad, la apuesta gastronómica promueve la revitalización de las tradiciones culinarias regionales y el consumo de sus productos. Bajo esta premisa, la oferta del restaurante se nutre de alimentos de proveedores locales que se encuentran en una isócrona de treinta minutos desde el hotel.

\subsection{Justificación del carácter innovador del Hotel Paisaje Vivood}

La presentación realizada en las líneas anteriores introduce la descripción analítica del caso y, también, pretende justificar su selección de acuerdo con los objetivos de investigación señalados. Se quiere, en todo caso, hacer evidente el hecho de que una propuesta como la del Vivood, que sitúa en el centro de su atractivo la tranquilidad y la exclusividad (por cierto, no admite niños) en un entorno de gran riqueza ecológica y valor paisajístico sobre el que se pretende una intervención mínima, se halla en las antípodas del modelo turístico masivo especializado en el residencialismo que predomina en la provincia de Alicante (Huete, 2009; Huete, 2016; Mantecón, 2008; Vera, 1987; Vera, 2005), cuyos rasgos definitorios, estudiados ya en profundidad, aluden a los graves impactos sobre el medio ambiente y a la desarticulación de la planificación territorial (Capdepón, 2016; Membrado Tena, 2011; Vera, 2005; Vera Rebollo e Ivars Baidal, 2003), a la proliferación de procesos de fragmentación social y de estrategias residenciales en las que se confunden los límites entre la migración y el turismo, con los consiguientes problemas que ello supone para la gestión municipal (Huete y Mantecón, 2010; Huete y Mantecón, 2011; Huete y Mantecón, 2012; Huete y Mantecón, 2013; Membrado Tena, 2015), a la produc- ción de lógicas en virtud de las cuales los municipios se estructuran económicamente en torno a la masiva construcción de viviendas publicitadas inicialmente como de uso turístico, impulsando dinámicas poco rentables para las sociedades locales en el medio y largo plazo (Perles Ribes, Ramón Rodríguez, Sevilla Jiménez y Moreno Izquierdo, 2016), a lo que habría que añadir la característica que quizá le concede una fisonomía más distintiva: el marcado desequilibrio existente entre, por un lado, la oferta reglada de alojamiento -vinculada sobre todo a hoteles y apartamentos registrados para su uso turístico- $y$, por otro, la oferta muy superior de alojamiento no reglado -ligada a viviendas particulares integradas en una economía inmobiliaria informal-.

El Vivood irrumpe en este contexto con una propuesta innovadora, es decir, creativa y rentable. La dimensión creativa no reside en el hecho de haber inventado el concepto Hotel Paisaje, pues existen desde hace tiempo hoteles paisaje en muchos lugares del mundo. Más bien, la novedad tiene que ver con su incrustación en un contexto posicionado desde hace cinco décadas sobre parámetros turísticos contrarios a los principios en los que se inspira el Vivood. Mayo no ha inventado algo nuevo, pero sí lo ha adaptado de forma novedosa a una realidad en la que no existía. El análisis empírico de la innovación ha mostrado que esta no suele asentarse tanto sobre cambios radicales como sobre transformaciones incrementales, y más en el ámbito del turismo (Williams, 2014). El Vivood, en este sentido, se revela como un ejemplo modélico.

Por lo que respecta a la rentabilidad que acompaña a la implementación de la propuesta, condición sin la cual no podría reconocerse la innovación, se muestran algunos datos. El coste de pasar una noche en una de las habitaciones del Vivood oscila, aproximadamente, entre los 120 y los 450 euros. Se subraya el hecho evidente de que el producto ofertado se dirige a un target con un poder adquisitivo más elevado que el del tipo medio de turista que se hospeda en el tipo medio de alojamiento de la Comunidad Valenciana donde, según los datos del Instituto Nacional de Estadística para 2015, se registraba un precio medio diario por habitación de 65,7 euros. Como se apuntaba más arriba, el hotel comenzó su actividad en junio de 2015, por eso al escribir estas líneas (septiembre de 2016) apenas se pueden hacer comparaciones interanuales. No obstante, ya es posible confrontar las cifras relativas a los meses estivales centrales: los porcentajes de ocupación en julio y agosto de 2015 fueron del $35 \%$ y el $78 \%$ respectivamente, ofreciendo 
alojamiento a 505 personas el primer mes y a 1.156 el segundo, mientras que en 2016 han sido del $84 \%$ y el $92 \%$, hospedando a 1.250 personas en julio y a 1.368 en agosto. Otro dato ilustrativo es el que se refiere a la antelación media con la que se reservaron las habitaciones: durante los siete meses de vida del hotel en 2015 fue de 13,8 días, pasando en los primeros ocho meses de 2016 a 26,1 días. Estos datos no permiten discernir con claridad la rentabilidad del hotel, pero invitan al optimismo.

\subsection{La relevancia del capital turístico situacional}

A continuación la imagen trazada del proyecto se problematiza al superponerla sobre un fondo en el que se identifican los principales desafíos que han jalonado su implementación. Las dificultades inherentes al reto planteado son innumerables. Las cuestiones relacionadas con la financiación siempre son determinantes, así como las complejas gestiones administrativas que han de emprenderse para obtener los permisos pertinentes. Las primeras se resolvieron en gran medida con la ayuda del fondo de inversión de Lanzadera; las segundas se agilizaron gracias a un concienzudo trabajo de seguimiento de los trámites en curso. La búsqueda de la localización idónea supuso una odisea de viajes entre Valencia y numerosos ayuntamientos de la provincia de Alicante, hasta que se optó por su emplazamiento actual. La inmediata identificación con la filosofía del proyecto y la comprensión de las ventajas que podía ofrecer al municipio predispuso muy favorablemente al ayuntamiento de Benimantell. Las explicaciones públicas proporcionadas desde la dirección del Vivood sirvieron igualmente para despejar dudas y obtener el respaldo de los residentes en el pueblo. Aunque, quizá, estos temas no son comparables con el hecho ya resaltado de que el proyecto Vivood desentonase tanto con las tendencias turísticas dominantes en la región. Al respecto, la ubicación definitiva en un paraje natural privilegiado situado a solo veinte minutos de Benidorm, el gigante turístico de la Costa Blanca, ha resultado decisiva.

Tras una primera mirada da la impresión de que no puede haber relación alguna entre el Vivood, un producto inequívocamente postfordista, y Benidorm, el rey del turismo fordista en el Mediterráneo. Sin embargo, en la práctica esa proximidad le ha permitido aprovechar un potente capital turístico situacional que se articula en un tejido de organizaciones y en una cultura muy favorables al desarrollo de negocios turísticos, facilitando así a los recién llegados la consolidación de provechosas relaciones comerciales. Ese capital turístico posee efectivamente un componente situacional muy particular pues se halla concentrado en Benidorm, un sistema turístico con entidad propia y tremendamente dinámico en el que la extensa oferta de alojamiento en vivienda privada se combina con una heterogénea oferta de ocio y con más de 40.000 plazas hoteleras que registran cada año alrededor de diez millones de pernoctaciones (Ivars Baidal, Rodríguez Sánchez y Vera Rebollo, 2012). Benidorm se distingue en la Comunidad Valenciana, y en la provincia de Alicante con especial nitidez, como una rara avis en un contexto mayor de residencialismo, casi hegemónico, donde la industria turística se ve fuertemente penetrada e instrumentalizada por la economía inmobiliaria. La proximidad a Benidorm sitúa al Vivood cerca de una densa red de proveedores, servicios de lavandería y limpieza, personal cualificado con formación en turismo y restauración, etc., y, también, de canales de comercialización y repercusión mediática que al cabo del año captan la atención de millones de personas. Es cierto que su localización aislada en la pendiente de un valle complica la frecuencia y el propio traslado de algunos suministros de consumo habitual, pero esas dificultades se están solucionando y quedan compensadas por la posibilidad real de acceso regular a los mismos. A su vez, una adecuada estrategia de marketing en las redes sociales desambigua la posición distintiva del Vivood respecto a la oferta mayoritaria de Benidorm y, en última instancia, el precio de las habitaciones ahuyenta a quienes pudieran confundirlo con una opción de alojamiento en la periferia benidormense a la que acudir cuando la planta hotelera de la ciudad cuelga el cartel de completo.

La aparición del Vivood fue percibida originalmente por el empresariado turístico de Benidorm entre, por un lado, la indiferencia ante un producto extraño y arriesgado que no representaba una competencia para nadie $y$, probablemente, al que se vaticinaba una corta vida y, por otro, la expectación por comprobar la evolución de una propuesta tan singular. Pasado el corte del primer año de vida, la indiferencia inicial se ha tornado en respeto y reconocimiento: de hecho, algunos empresarios del sector turístico benidormense se han hospedado en el hotel para conocerlo de primera mano e intercambiar opiniones.

\subsection{Riesgos y amenazas futuras}

Se comentan ahora algunos de los riesgos que pueden perturbar el devenir del proyecto. Para ordenar la explicación se distingue entre riesgos potenciales motivados por dinámicas internas y externas.

Sobre los primeros se apuntan dos cuestiones. Desde la apertura del hotel la estancia de los huéspedes 
se halla estancada alrededor de una media de 1,4 noches. La percepción del Vivood como un lugar en el que realizar una estancia muy breve, de una noche, y ocasionalmente de dos, se asocia a la imagen que el propio hotel proyecta de rincón especial reservado para disfrutar de momentos puntuales y excepcionales. Pero con estancias tan cortas es difícil rentabilizar los esfuerzos invertidos. La respuesta de la dirección para evitar que este asunto se vuelva crónico se orienta hacia la diversificación de la oferta complementaria al alojamiento: organización de paseos y excursiones, sesiones especiales de astronomía, trekking nocturno o noche de las velas, entre otras que, junto a promociones especiales, animen a prolongar la duración de las estancias. Una segunda cuestión, también relativa a la necesidad de mejorar la rentabilidad, es consustancial al objetivo reconocido de ampliar la capacidad de alojamiento aumentando el número de suites, esto es, de módulos construidos, de los veinticinco actuales a una cifra aproximada de cuarenta. Realmente, es muy complicado hacer rentable un negocio de estas características con el rendimiento que se puede obtener de únicamente veinticinco habitaciones. Los riesgos son claros, en tanto que la posibilidad de disfrutar de un paisaje privilegiado en unas instalaciones exclusivas, la esencia definitoria del Vivood, puede alterarse al aumentar el flujo de personas que circulan por los espacios comunes. Sin embargo, este parece el problema menos difícil de resolver si se sopesan los recursos con los que la empresa cuenta, los cuales se materializan en la capacidad real para hacer un ejercicio de acupuntura arquitectónica sobre una parcela que, al fin y al cabo, consta de $84.000 \mathrm{~m}^{2}$.

En cambio, las amenazas más inquietantes, debido a la complejidad que entraña formular una respuesta efectiva a las mismas, se vislumbran en el medio y largo plazo a propósito de dinámicas exógenas. El Hotel Paisaje Vivood se construyó en un periodo de colapso de la industria del ladrillo. Algo fácil de apreciar en la Comunidad Valenciana si se tiene en cuenta la virulencia con la que se manifestó el tsunami urbanizador en esta región durante los años anteriores al estallido de la burbuja inmobiliaria (Gaja i Díaz, 2008). Emprender un negocio como el Vivood en el marco de una profunda crisis económica implica dificultades, pero también algunas ventajas que podrían desaparecer ante una futura revitalización de la expansión urbanística.

En 2008 la Conselleria de Infraestructuras y Transportes de la Generalitat Valenciana incluía dentro del plan de carreteras la transformación de la comarcal CV-70 que une Alcoy con Benidorm en una vía rápida o au- tovía que acortaría el tiempo dedicado a completar el trayecto entre ambas localidades. El nuevo vial atravesaría el Valle de Guadalest a la altura de Benimantell e incluía la construcción de un túnel para salvar el Puerto de Ares, en el municipio de Confrides. La noticia provocó la movilización en contra del proyecto de los habitantes del valle, más aún si se considera que las sierras que lo conforman estaban reconocidas como "Lugares de Interés Comunitario" y que poco antes un grupo de expertos de la Universidad de Alicante habían propuesto su declaración como "Reserva de la Biosfera". Sin embargo, desde los municipios de la costa y la segunda línea, como La Nucía (a medio camino entre Benimantell y Benidorm), el proyecto se veía con mejores ojos a tenor de las declaraciones que hacían algunos de sus representantes políticos. En septiembre de 2009 el proyecto era presentado por el conseller de Infraestructuras y Transportes en el Ayuntamiento de Alcoy. Sin embargo, en los presupuestos de la Generalitat para 2011 desaparecían las partidas correspondientes a la ejecución de las obras debido a la falta de fondos y la idea quedó aparcada indefinidamente.

La conservación del patrimonio ecológico del Valle de Guadalest se relaciona con su ubicación en una franja interior cuyos accesos desde la costa no han sido fáciles. Durante mucho tiempo esa dificultad ha servido de profilaxis ante la posibilidad de convertirse en un objetivo prioritario para los intereses inmobiliarios. Precisamente, las obras de mejora y ampliación de la carretera CV-70 en el tramo que conecta Benimantell con el litoral se acometieron hace una década, justamente cuando se produjo el crac urbanístico que detuvo la fiebre del ladrillo. En fin, no parece descabellado pensar que la modernización de la red de comunicaciones entre el valle y la costa, ya realizada, pueda animar en los próximos años el advenimiento de propuestas urbanizadoras consistentes en la construcción de diseminados de chalets o macroproyectos residenciales. Tal y como ha ocurrido desde finales de los noventa en otros lugares cercanos, la saturación y degradación de la primera y segunda línea de costa ha motivado la expansión de las dinámicas residenciales, reproducidas una y otra vez en el litoral, hacia los municipios del interior. Sin minusvalorar la predisposición de los ciudadanos a preservar la riqueza ecológica del valle que habitan, está por ver si en todos los municipios que configuran este sistema ecológico serán capaces de resistirse a la llamada del dinero fácil, pues con que solo uno decidiera poner sus recursos naturales al servicio de la economía inmobiliaria la perspectiva paisajística actual quedaría perturbada radicalmente. Desde este punto de vista, parece plausible 
una nueva confluencia entre las élites económicas y políticas constituidas en torno al negocio urbanístico para favorecer la implantación de grandes infraestructuras que acorten el tiempo de desplazamiento entre la costa y los espacios mejor conservados del interior. Así, en octubre de 2015, y tras el cambio de gobierno autonómico, un grupo de empresarios aprovechó una reunión celebrada en la Cámara de Comercio e Industria de Alcoy con el conseller de Economía Sostenible, Sectores Productivos, Comercio y Trabajo para solicitar, una vez más esgrimiendo la excusa del desarrollo turístico, que se recupere el plan original de construcción de una autovía entre Alcoy y Benidorm atravesando el Valle de Guadalest.

La respuesta ante esta amenaza es compleja, pues exige una intensa colaboración entre grupos de ciudadanos y ayuntamientos a escala supramunicipal. Al mismo tiempo, ello requiere políticos locales con la suficiente altura de miras como para trascender los intereses particulares de cada municipio, llegar a acuerdos que tengan como objetivo preservar la riqueza ecológica existente en el Valle de Guadalest y promover iniciativas empresariales sostenibles. Por el momento, no hay razones que animen especialmente al optimismo, cuando localismos trasnochados y cuestiones identitarias mal entendidas impiden pensar el valle como una única entidad geográfica coherente e integrada al tiempo que entorpecen el diseño de estrategias de colaboración sobre cuestiones de menor envergadura. De un modo u otro, la respuesta más realista pasará por la conformación de un sistema de relaciones económicas que articule el tejido empresarial alrededor de negocios sostenibles, cuya existencia esté supeditada a la protección del patrimonio ecológico: restaurantes que oferten platos tradicionales de la comarca, albergues, tiendas que vendan productos locales, un posible centro de BTT (bicicletas todo terreno) u otras actividades deportivas y recreativas que no supongan una agresión sobre el entorno. En fin, empresas dependientes de la afluencia de turistas y excursionistas atraídos por la riqueza natural y la ausencia de las huellas que en otros lugares ha dejado la voracidad urbanística. Las conciencias de los habitantes, pero también sus negocios, han de hallarse vinculados a la preservación del medio. En este sentido, el éxito de un proyecto innovador como el del Vivood puede actuar de referente, y más vale que así lo haga pues su futuro depende de ello.

\section{CONCLUSIONES}

El proyecto Vivood se constituye en un caso paradigmático donde el paisaje mediterráneo es ensalza- do como un recurso turístico en sí mismo, susceptible de ser consumido a través de la mirada turística, y no convertido en un medio al servicio del negocio inmobiliario, como reiteradamente ha sucedido bajo el pretexto de un supuesto desarrollo turístico (Huete y Tros-de-Ilarduya, 2011; Mantecón, 2010).

La instrumentalización de la economía turística por la inmobiliaria, y su ilustrativa plasmación en la provincia de Alicante en la desproporcionada expansión de las dinámicas residenciales, no impide todavía el establecimiento de sinergias beneficiosas entre actores que pertenezcan al sistema turístico, aunque estas se fragüen entre vecinos tan diferentes como el Vivood y la industria de ocio y turismo de Benidorm. Sobreponiéndose a un entorno más amplio de residencialismo exacerbado, tanto el Vivood como Benidorm representan, cada uno a su manera, propuestas innovadoras originadas y desplegadas desde el sector turístico, en sentido estricto. En las líneas anteriores se han comentado los beneficios que obtiene el pequeño y pintoresco recién llegado establecimiento hotelero del colosal referente del turismo mediterráneo, inmerso en un proceso de renovación constante desde hace más de medio siglo. Falta por comprobar si los empresarios de Benidorm son capaces de entender cómo un vecino minúsculo como el Vivood también puede reportarles beneficios. Sobre este asunto interesa recuperar el artículo periodístico de Rivalland: The other side of Benidorm, publicado en el diario británico The Times el 21 de noviembre de 2015. En él se utiliza el caso del Vivood Landscape Hotel para desmitificar la imagen de Benidorm y de su entorno inmediato como un espacio carente de atractivos para quienes se interesan por opciones de alojamiento y ocio alternativas al sol y playa en paquetes estandarizados de bajo coste. Obviamente, no se sugiere a estas alturas reposicionar a Benidorm, pues sería absurdo además de poco inteligente desde el punto de vista de la estrategia empresarial, pero igualmente podría resultar positivo que poco a poco se introdujeran matices mediante los que se identificara a esta ciudad dentro de un entramado geográfico de ofertas de ocio un poco más amplio. Quizá, también el empresariado de Benidorm saldría ganando.

Los desafíos a los que se enfrenta el Vivood en el futuro no son pequeños. Como se ha argumentado, los más difíciles de resolver provienen de amenazas externas asociadas a la proyección de lógicas de actuación, ajenas al sistema turístico propiamente dicho, impulsadas por la economía inmobiliaria en la provincia de Alicante desde hace décadas. Tras la 
interrupción de la expansión urbanística entre 2007 y 2008 , una consecuencia observable desde el punto de vista de la gestión turística es la drástica reducción del margen de maniobra disponible para redirigir las opciones futuras de desarrollo local. La formulación de respuestas coordinadas en un nivel supramunicipal y sustentadas en la promoción y defensa de una economía local orientada por firmes principios de sostenibilidad y eficiencia ecológica se ha discutido como una opción viable y difícil.

El incipiente éxito cosechado por el Vivood y por otros proyectos distintivos, como el Asia Gardens (un lujoso complejo hotelero de cinco estrellas, con 312 habitaciones, tematizado con motivos balineses) ubicado en Finestrat, a 13 kilómetros de Benidorm, prueban que es posible aprovechar el capital turístico situacional existente en un contexto de turismo de masas maduro, como el benidormense, para generar una oferta especializada basada en la creación de un producto exclusivo y diferenciado. Si se consigue incorporar ese valor añadido (no necesariamente sustentado en aspectos materiales) a un entorno que ya posee una dilatada experiencia con el turismo de sol y playa, también se puede multiplicar el precio medio del coste de las estancias.

La adecuada interacción entre unas aptitudes personales y un medio social proclives a la innovación se reconocen como claves fundamentales. Se trata, finalmente, de poseer la imaginación para diseñar un producto innovador y la capacidad de captar los recursos que hacen falta para convertirlo en realidad. No son elementos abstractos sino factores reales que aparecen como consecuencia de la combinación de ingredientes tradicionales: pasión irreductible, formación sólida e interdisciplinar, investigación ordenada, espíritu de sacrificio, trabajo constante, grandes dosis de paciencia y resistencia al fracaso, todo ello sazonado con unas gotas de talento y creatividad en un medio socioeconómico, político y cultural que sepa apreciar el resultado. La ejecución de la receta no es sencilla, pero, a pesar del contenido de los mensajes que emiten habitualmente los medios de comunicación y la publicidad, lo cierto es que pocas cosas significativas se consiguen sin la inversión de un gran esfuerzo.

\section{BIBLIOGRAFÍA}

Capdepón, M. (2016). Conflictos ambientales derivados de la urbanización turístico-residencial. Un caso aplicado al litoral alicantino. Boletín de la Asociación de Geógrafos Españoles, 71, pp. 31-57.

Cohen, E. (2004). Contemporary Tourism. Diversity and Change. Kidlington: Pergamon.

Cohen, E. (2005). Principales tendencias en el turismo contemporáneo. Política y Sociedad, 42, 1, pp. 11-24.

Gaja i Díaz, F. (2008). El "tsunami urbanizador" en el litoral mediterráneo. El ciclo de hiperproducción inmobiliaria 1996 2006. Scripta Nova. Revista Electrónica de Geografía y Ciencias Sociales, XII, 270, 66.

Hall, C. M. y Williams, A. M. (2008). Tourism and Innovation. London: Routledge.

Huete, R. (2009). Turistas que llegan para quedarse. Una explicación sociológica sobre la movilidad residencial. Alicante: Publicaciones de la Universidad de Alicante.

Huete, R. (2016). La vertiente residencial del turismo. Canelobre, 66, pp. 125-133.
Huete, R. y Mantecón, A. (2010). Los límites entre el turismo y la migración residencial. Una tipología. Papers. Revista de Sociologia, 95, 3, pp. 781-801. https:// doi.org/10.5565/rev/papers/v95n3.95

Huete, R. y Mantecón, A. (2011). Más allá del turismo: movilidad residencial europea y nuevos núcleos urbanos. Boletín de la Asociación de Geógrafos Españoles, 56, pp. 111-128.

Huete, R. y Mantecón, A. (2012). Residential tourism or lifestyle migration. Social problems linked to the non-definition of the situation. En Moufakkir, O. y Burns, P. (eds.). Controversies in Tourism. Wallingford: CABI, pp. 160-173. https://doi. org/10.1079/9781845938130.0160

Huete, R. y Mantecón, A. (2013). La migración residencial de noreuropeos en España. Convergencia. Revista de Ciencias Sociales, 61, pp. 219-245.

Huete, R. y Tros-de-Ilarduya, M. (2011). La valorización de los espacios naturales como pretexto para el desarrollo urbanístico en el sudeste de España. Proyección, V, pp. 102-124.
Ivars Baidal, J., Rodríguez Sánchez, I. y Vera Rebollo, F. (2012). The evolution of mass tourism destinations: new approaches beyond deterministic models in Benidorm (Spain). Tourism Management, 34, pp. 184-195. https://doi. org/10.1016/j.tourman.2012.04.009

Mantecón, A. (2008). La experiencia del turismo. Un estudio sociológico sobre el proceso turístico-residencial. Barcelona: Icaria.

Mantecón, A. (2010). Tourist modernisation and social legitimation in Spain. International Journal of Tourism Research, 12, 5, pp. 617-626. https://doi. org/10.1002/jtr.784

Membrado Tena, J. C. (2011). Cartografía del urbanismo expansivo valenciano según SIOSE. En: Gozálvez Pérez, V. y Marco Molina, J. A. (eds.). Urbanismo expansivo. De la utopía a la realidad. Madrid: Asociación de Geógrafos Españoles, pp. 427-438.

Membrado Tena, J. C. (2013). Sunny Spain: migrantes del sol y urbanismo expansivo en el litoral mediterráneo español. Ciudad y Territorio. Estudios Territoriales, XLV, 178, pp. 687-708. 
Membrado Tena, J. C. (2014). La costa dels jubilats: la migració nord-europea de persones retirades a la Costa Blanca. Mètode. Revista de difusió de la investigació de la Universitat de València, 81, pp. 62-71. https://doi.org/10.7203/metode.81.3111

Membrado Tena, J. C. (2015). Migración residencial y urbanismo expansivo en el Mediterráneo español. Cuadernos de Turismo, 35, pp. 259-285.

Monette, D. R., Sullivan, T. J. y DeJong, C. R (2005). Applied Social Research. A Tool for the Human Services. Belmont, CA: Thomson-Brooks/Cole.

Perles Ribes, J. F., Ramón Rodríguez, A. B. Sevilla Jiménez, M. y Moreno Izquierdo, L. (2016). Unemployment effects of economic crises on hotel and residential tourism destinations: The case of Spain. Tourism Management, 54, pp. 356-368. https://doi.org/10.1016/j.tourman.2015.12.002
Rivalland, M. (2015, 21 de noviembre). The other side of Benidorm. The Times (sección Viajes), pp. 26-27.

Rodríguez, I. (2016). Innovación y turismo: una aproximación inicial al tema. Canelobre, 66, pp. 435-443.

Rodríguez, I., Williams, A. M. y Hall, C. M. (2014). Tourism innovation policy: implementation and outcomes. Annals of Tourism Research, 49, pp. 76-93. https://doi.org/10.1016/j.annals.2014.08.004

Roger, A. (1997). Court traité du paysage. París: Gallimard.

Vera, F. (1987). Turismo y urbanización en el litoral alicantino. Alicante: Instituto de Cultura Juan Gil Albert.

Vera, F. (2005). El auge de la función residencial en destinos turísticos del litoral mediterráneo: entre el crecimiento y la renovación. Papers de Turisme, 37/38, pp. 95-114.
Vera Rebollo, F. e Ivars Baidal, J. (2003). Measuring sustainability in a mass tourist destination: pressures, perceptions and policy responses in Torrevieja, Spain. Journal of Sustainable Tourism, 11, 2-3, pp. 181-203. https://doi. org/10.1080/09669580308667202

Williams, A. M. (2014). Tourism Innovation: Products, Processes, and People. En: Lew, A. A., Hall, C. M. y Williams, A. M. (eds.). The Wiley Blackwell Companion to Tourism. Oxford: Wiley-Blackwell, pp. 168-178. https://doi. org/10.1002/9781118474648.ch13

\section{Otros recursos}

Vivood hotel paisaje. [En línea]. Disponible en: https://www.vivood.com 